\title{
About the Optimality of Oscillations in Non-Lipschitz Coefficients for Strictly Hyperbolic Equations
}

\author{
FUMIHIKO HIROSAWA - MICHAEL REISSIG
}

Dedicated to Professor Masaru Yamaguchi on the occasion of his sixtieth birthday

\begin{abstract}
In the present paper we explain the classification of oscillations and its relation to the loss of derivatives for a homogeneous hyperbolic operator of second order. In this way we answer the open question if the assumptions to get $C^{\infty}$ well posedness for weakly hyperbolic Cauchy problems or for strictly hyperbolic Cauchy problems with non-Lipschitz coefficients are optimal.
\end{abstract}

Mathematics Subject Classification (2000): 35L15 (primary), 35L10, 35A05 (secondary).

\section{1. - Introduction}

We are interested in the backward strictly hyperbolic Cauchy problem

$$
\begin{aligned}
\left(D_{t}^{2}+2 b(t) D_{x t}^{2}-a(t)^{2} D_{x}^{2}\right) u(t, x) & =0, \quad(t, x) \in[0, T] \times \mathbb{R}, \\
u(T, x)=\varphi(x), u_{t}(T, x) & =\psi(x), \quad x \in \mathbb{R},
\end{aligned}
$$

where $D=-i \partial$, and the data $(\varphi, \psi) \in H^{s+1} \times H^{s}$ are prescribed on the hyperplane $t=T$. Later we will formulate conditions for $a=a(t)$ and $b=b(t)$ (see Theorems 1 to 3 ) which imply that the Cauchy problem is well-posed in classes of Sobolev spaces on any interval $\left[T_{1}, T\right], T_{1}>0$, without any loss of regularity. For this reason we assume $T>0$ small without loss of generality. The coefficients $a=a(t)$ and $b=b(t)$ are assumed to be bounded on the interval $[0, T]$. The strict hyperbolicity assumption means

$$
b(t)^{2}+a(t)^{2}>0 \quad \text { on } \quad[0, T]
$$

Pervenuto alla Redazione il 28 gennaio 2004 e in forma definitiva il 28 maggio 2004. 
If we are interested in solutions which are valued in Sobolev spaces, then we have to assume more regularity to the coefficients than $\bigcap_{\alpha \in(0,1)} C^{\alpha}([0, T])$ (see the counter-examples from [5]).

Let us suppose that the coefficients belong to the class of Lipschitz continuous functions $\operatorname{Lip}([0, T])$, then under the condition (1.2) one can prove the well-posedness of the Cauchy problem (1.1) in all Sobolev classes $H^{s}, s \geq 0$. Moreover, the following energy inequality holds:

$$
\left\|\left(\partial_{x} u, \partial_{t} u\right)(t, \cdot)\right\|_{H^{s}} \leq C\left\|\left(\varphi^{\prime}(\cdot), \psi(\cdot)\right)\right\|_{H^{s}} \text { for } 0 \leq t<T .
$$

The energy inequality tells us, that the energy solution of the backward Cauchy problem can be continued up to $t=0$. But the Lipschitz condition is not necessary for having solutions with a finite energy. There are at least two ways to weaken this condition.

a) Global condition.

This idea goes back to [3]. The authors supposed the so-called LogLipproperty for the coefficients. This means $\left|a\left(t_{1}\right)-a\left(t_{2}\right)\right| \leq C\left|t_{1}-t_{2}\right||\log | t_{1}-t_{2}||$ for all $t_{1}, t_{2} \in[0, T]$ with $t_{1} \neq t_{2}$; we denote the class of such functions by $\log \operatorname{Lip}([0, T])$.

From the results of [1] we know that $a, b \in \operatorname{LogLip}([0, T])$ implies an energy estimate like (1.3) with a finite loss of derivatives, that is,

$$
\left\|\left(\partial_{x} u, \partial_{t} u\right)(t, \cdot)\right\|_{H^{s-s_{0}}} \leq C\left\|\left(\varphi^{\prime}(\cdot), \psi(\cdot)\right)\right\|_{H^{s}} \text { for } 0 \leq t<T,
$$

where $s_{0} \geq 0$ describes the so-called loss of derivatives.

REMARK 1. The authors expect the following connection between the regularity of coefficients and the loss of derivatives (see also [10]): suppose for (1.1) that the coefficients $a, b$ belong to $\log ^{\gamma} \operatorname{Lip}([0, T])$, which denotes the class of functions $a$ satisfying $\left|a\left(t_{1}\right)-a\left(t_{2}\right)\right| \leq C\left|t_{1}-t_{2}\right||\log | t_{1}-\left.t_{2}\right|^{\gamma}$ for all $t_{1}, t_{2} \in[0, T]$ with $t_{1} \neq t_{2}$, and with $\gamma \geq 0$, and that the coefficients satisfy (1.2), then the energy estimates (1.4) hold with

- $s_{0}=0$ if $\gamma=0$;

- $s_{0}$ is arbitrary small and positive if $\gamma \in(0,1)$;

- $s_{0}$ is positive if $\gamma=1$;

- there is no positive constant $s_{0}$ if $\gamma>1$ (infinite loss of derivatives).

Thus, the counter-example from [6] implies the statement for $\gamma>1$. The approach from [1] should give the statement for $\gamma \in[0,1]$.

b) LOCAL CONDITION.

A second possibility to weaken the Lip-property with respect to $t$ goes back to [4]. Under the assumptions (1.2) and

$$
b(t) \equiv 0, a \in L_{\infty}([0, T]) \cap C^{1}((0, T]),\left|t a^{\prime}(t)\right| \leq C \text { for } t \in(0, T]
$$


one can show that the energy inequality (1.4) holds for all $t \in[0, T]$ with a non-negative constant $s_{0}$.

If we suppose more regularity for $a$, let us say, $a \in L_{\infty}([0, T]) \cap C^{2}((0, T])$, then a refined classification of oscillations of the coefficient can be introduced.

Definition 1. We call the oscillating behaviour of the function $a(t) \in$ $C^{2}((0, T])$ satisfying for positive constants $a_{0}, a_{1}$ and $R$ the conditions $a_{0} \leq$ $a(t) \leq a_{1}$, and

$$
\left|a^{(k)}(t)\right| \leq\left(R t^{-1}\left(\log t^{-1}\right)^{\gamma}\right)^{k}, \quad k=1,2, \quad \text { for } t \in(0, T],
$$

- very slow oscillation if $\gamma=0$;

- slow oscillation if $\gamma \in(0,1)$;

- fast oscillation if $\gamma=1$;

- very fast oscillation if condition (1.6) is not satisfied for $\gamma=1$.

EXAMPLE 1. If $a=a(t)=2+\sin \left(\log t^{-1}\right)^{\alpha}$, then the oscillations produced by the $\sin$ term are very slow (slow, fast, very fast) if $\alpha \leq 1,(1<\alpha<2$, $\alpha=2, \alpha>2$ ).

Then one can prove the following result (see [5], [7] and [13]).

Proposition 1. Let us suppose for (1.1) that $b \equiv 0$ and a satisfies the conditions (1.2), (1.5) and (1.6). Then the energy estimates

$$
\left\|\left(\partial_{x} u, \partial_{t} u\right)(t, \cdot)\right\|_{H^{s-s_{0}}} \leq C\left\|\left(\varphi^{\prime}(\cdot), \psi(\cdot)\right)\right\|_{H^{s}} \text { for } 0 \leq t<T
$$

hold with

- $s_{0}=0$ if $\gamma=0$;

- $s_{0}$ is arbitrary small and positive if $\gamma \in(0,1)$;

- $s_{0}$ is positive if $\gamma=1$;

- there is no positive constant $s_{0}$ if $\gamma>1$ (infinite loss of derivatives).

REMARK 2. We supposed the boundedness of the coefficients in the present paper for simplicity. However, the condition $a \in L_{\infty}([0, T])$ is not necessary in general. Indeed, one can prove similar results if $a$ is estimated by suitable unbounded functions near $t=0$ (see [4], [7] and [13]).

REMARK 3. There is no relation between these both possibilities a) and b) to weaken the Lipschitz property. Each possibility has its own meaning.

REMARK 4. There arises after the results of [4] and [5] the question whether there is something between the conditions (1.2), (1.5) and (1.2), (1.6). The paper [8] is devoted to the model Cauchy problem

$$
u_{t t}-a(t, x) \triangle u(t, x)=0, u(T, x)=\varphi(x), u_{t}(T, x)=\psi(x),
$$

where $a \in L_{\infty}\left([0, T], B^{\infty}\left(\mathbb{R}^{n}\right)\right)$ and $a_{0} \leq a(t, x)$ with a positive constant $a_{0}$. It is shown that there exists a $C^{1, \beta}$-version of Proposition 1 with $\beta \in(0,1)$, 
where $C^{1, \beta}$ denotes the usual Hölder space of degree $\beta+1$. This means, if $a \in C^{1, \beta}((0, T])$ with respect to $t$, then suitable conditions for $a$ guarantee the same relation between the classification of oscillations and the loss of derivatives (in the case $\gamma \in[0,1]$ ) which is described in Proposition 1.

All results explained up to now and basing on the local condition assume $b(t) \equiv 0$ for the starting equation from (1.1). It seems to be natural to ask if we can prove the statement from Proposition 1 without the condition $b \equiv 0$. In the present paper we will show, that in general this kind of generalization does not hold.

THEOREM 1. Let us consider the strictly hyperbolic Cauchy problem (1.1). There exist coefficients $a$ and $b$ from $C^{2}((0, T])$ which satisfy the conditions

- $a_{0} \leq a(t) \leq a_{1}, b_{0} \leq b(t) \leq b_{1}$;

- $\left|a^{\prime}(t)\right|^{2}+\left|a^{\prime \prime}(t)\right| \leq\left(R t^{-1}\left(\log t^{-1}\right)^{\gamma}\right)^{2}$;

- $\left|b^{\prime}(t)\right|^{2}+\left|b^{\prime \prime}(t)\right| \leq\left(R t^{-1}\left(\log t^{-1}\right)^{\gamma}\right)^{2}$

with positive constants $a_{0}, a_{1}, b_{0}, b_{1}, R$ and with an arbitrary small positive $\gamma$ such that the Cauchy problem (1.1) is not $C^{\infty}$ well-posed, that is, the energy inequality

$$
\left\|\left(\partial_{x} u, \partial_{t} u\right)(t, \cdot)\right\|_{H^{s-s_{0}}} \leq C\left\|\left(\varphi^{\prime}(\cdot), \psi(\cdot)\right)\right\|_{H^{s}} \text { for } 0 \leq t<T
$$

does not hold for any positive $s_{0}$ with a positive constant $C$ independent of the data $(\varphi, \psi)$.

REMARK 5. The statement of this theorem explains that in the case that the term $2 b(t) D_{x t}^{2} u$ arises the relation between the type of oscillations and the loss of derivatives from Proposition 1 will change. If both coefficients $a$ and $b$ contain slow oscillations in the language of Definition 1, an infinite loss of derivatives can appear. This infinite loss of derivatives follows from the inequality

$$
\xi^{2}|\hat{u}(t, \xi)|^{2}+\left|\hat{u}_{t}(t, \xi)\right|^{2} \geq C \exp \left((\log |\xi|)^{\gamma+1}\right)\left(\xi^{2}|\hat{u}(T, \xi)|^{2}+\left|\hat{u}_{t}(T, \xi)\right|^{2}\right)
$$

uniformly for all $t \in[0, T]$ with a positive $\gamma$, where $\hat{u}$ denotes the Fourier transform of $u$ with respect to $x$. This formula follows from (3.19) from the proof to Theorem 1. But this implies that in general we cannot expect a solution from $C^{1}\left([0, T], D^{\prime}(\mathbb{R})\right)$.

REMARK 6. In the recent paper [2] the following strictly hyperbolic Cauchy problem is studied:

$$
\begin{gathered}
\left(D_{t}^{m}-\sum_{j=0}^{m-1} \sum_{|\alpha| \leq m-j} a_{j \alpha}(t, x) D_{t}^{j} D_{x}^{\alpha}\right) u(t, x)=0, \quad(t, x) \in[0, T] \times \mathbb{R}^{n}, \\
\left(\partial_{t}^{j} u\right)(T, x)=\phi_{j}(x), \quad j=0,1, \ldots, m-1, \quad x \in \mathbb{R}^{n},
\end{gathered}
$$


under the assumptions $t \partial_{t} a_{j \alpha}(t, x) \in \mathcal{B}^{0}\left((0, T], \mathcal{B}^{\infty}\left(\mathbb{R}^{n}\right)\right)$ for any $j$ and $\alpha$. Under these assumptions a finite loss of derivatives is proved. The statement of Theorem 1 shows that this assumption with respect to $t$ is optimal for the finite derivative loss. We expect that the statements of Theorems 2 and 3 which will be introduced later (see Sections 2 and 4) can be generalized to the $x$-dependent case.

\section{2. - Review of the proof of Proposition 1}

Here we will explain the main steps which allow to understand the statement of Proposition 1. The application of the partial Fourier transformation to the differential equation from (1.1) gives

$$
\left(D_{t}^{2}+2 b(t) \xi D_{t}-a(t)^{2} \xi^{2}\right) v(t, \xi)=0, \quad v(t, \xi)=\hat{u}(t, \xi)
$$

Setting $V(t, \xi):=\left(\xi v(t, \xi), D_{t} v(t, \xi)\right)^{T}$ the equation (2.1) can be transformed to the following first order system

$$
\left.\left(D_{t}-\left(\begin{array}{cc}
0 & \xi \\
a(t)^{2} \xi & -2 b(t) \xi
\end{array}\right)\right)\right) V(t, \xi)=0 .
$$

By the aid of the characteristic roots

$$
\tau_{1 / 2}=\tau_{1 / 2}(t):=-b(t) \mp \sqrt{a(t)^{2}+b(t)^{2}}
$$

let us define the matrix

$$
M(t)=\left(\begin{array}{cc}
1 & 1 \\
\tau_{1}(t) & \tau_{2}(t)
\end{array}\right)^{-1}
$$

Substituting $V_{0}(t, \xi):=M(t) V(t, \xi)$ some calculations transform the above system into the first-order system

$$
\left(D_{t}-\mathcal{D}+B\right) V_{0}(t, \xi)=0,
$$

where

$$
\mathcal{D}=\mathcal{D}(t, \xi):=\left(\begin{array}{cc}
\tau_{1} \xi & 0 \\
0 & \tau_{2} \xi
\end{array}\right) \quad \text { and } \quad B=B(t):=\frac{1}{\tau_{2}-\tau_{1}}\left(\begin{array}{cc}
-D_{t} \tau_{1} & -D_{t} \tau_{2} \\
D_{t} \tau_{1} & D_{t} \tau_{2}
\end{array}\right)
$$

This is the first step of diagonalization procedure. One can carry out further steps of diagonalization procedure, but the diagonal part of $B$; is unchanged 
(see [11]). During the construction of fundamental solution to (2.2) the influence of the integrals

$$
\text { (2.3) } \mp \int_{t}^{T} \frac{\frac{d}{d s} \tau_{1 / 2}(s)}{\tau_{2}(s)-\tau_{1}(s)} d s=\int_{t}^{T} \frac{\frac{d}{d s} \sqrt{a^{2}(s)+b^{2}(s)}}{2 \sqrt{a^{2}(s)+b^{2}(s)}} d s \pm \int_{t}^{T} \frac{\frac{d}{d s} b(s)}{2 \sqrt{a^{2}(s)+b^{2}(s)}} d s,
$$

which are integrals of the diagonal part of $B$, plays an important role. One should understand this influence for all $t \in\left[t_{\xi}, T\right]$, where $t_{\xi}$ is defined by $t_{\xi}\langle\xi\rangle=N(\log \langle\xi\rangle)^{\gamma}$ (see [11] or the proof of Theorem 1).

The first integral of (2.3) has no influence on the loss of derivatives because of (1.2). The trouble comes from the second one which disappears if $b(t) \equiv 0$. Thus in the case $b(t) \equiv 0$ both integrals do not produce a loss of derivatives. Carrying out the second step of diagonalization makes it possible to show that the new diagonal terms have an influence on the loss of derivatives which depends on the properties of $a$ which are described in Proposition 1. The proof of Theorem 1 bases on the fact that the second of the above integrals can produce an infinite loss of derivatives. But this is not always the case. If we suppose e.g. $\left|t b^{\prime}(t)\right| \leq C$ for $t \in(0, T]$, then the second integral produces only a finite loss of derivatives.

Carrying out the second step of perfect diagonalization the approach from [11] gives the following result.

THEOREM 2. Let us consider the Cauchy problem (1.1). If the coefficients satisfy

- $a, b \in L_{\infty}([0, T]) \cap C^{2}((0, T])$;

- $a(t)^{2}+b(t)^{2} \geq C>0$ on $(0, T]$

- $\left|a^{\prime}(t)\right|^{2}+\left|a^{\prime \prime}(t)\right| \leq\left(R t^{-1} \log t^{-1}\right)^{2}$ on $(0, T]$;

- $\left|b^{\prime}(t)\right|^{2}+\left|b^{\prime \prime}(t)\right| \leq\left(R t^{-1}\right)^{2}$ on $(0, T]$

with a positive constant $R$, then the Cauchy problem (1.1) is $C^{\infty}$ well-posed, that is, the energy inequality

$$
\left\|\left(\partial_{x} u, \partial_{t} u\right)(t, \cdot)\right\|_{H^{s-s_{0}}} \leq C\left\|\left(\varphi^{\prime}(\cdot), \psi(\cdot)\right)\right\|_{H^{s}}
$$

holds with a positive $s_{0}$ and with a positive constant $C$ independent of the data $(\varphi, \psi)$.

In Section 4 we will describe other assumptions to the coefficients $a$ and $b$ under which we can prove the same statement about the finite loss of derivatives.

\section{3. - Proof of Theorem 1}

\section{1. - Reduction to a suitable first order system}

We will consider the Cauchy problem

$$
\left(D_{t}^{2}+2 b(t) \xi D_{t}-a(t)^{2} \xi^{2}\right) v(t, \xi)=0, v(T, \xi)=\hat{\varphi}(\xi), v_{t}(T, \xi)=\hat{\psi}(\xi),
$$


which is obtained from (1.1) by partial Fourier transformation with respect to $x$. After the transformation

$$
w(t, \xi):=\exp \left(-i \xi \int_{t}^{T} b(s) d s\right) v(t, \xi)
$$

the Cauchy problem (3.1) can be rewritten as follows:

$$
\begin{gathered}
\left(D_{t}^{2}-c(t)^{2} \xi^{2}-\left(D_{t} b(t)\right) \xi\right) w(t, \xi)=0, \\
w(T, \xi)=\hat{\varphi}(\xi), \quad w_{t}(T, \xi)=i b(T) \xi \hat{\varphi}(\xi)+\hat{\psi}(\xi),
\end{gathered}
$$

where $c(t):=\sqrt{a(t)^{2}+b(t)^{2}}$. Setting $W_{0}:=\left(D_{t} w, c(t) \xi w\right)^{T}$ we obtain from (3.2) the following first order system for $W_{0}$ :

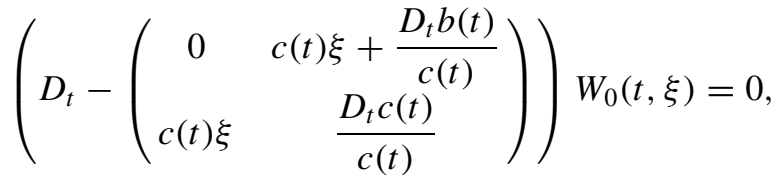

$$
\begin{aligned}
& W_{0}(T, \xi)=(b(T) \xi \hat{\varphi}(\xi)-i \hat{\psi}(\xi), c(T) \xi \hat{\varphi}(\xi)) .
\end{aligned}
$$

Let us carry out the first step of diagonalization procedure. We define the matrix

$$
M_{1}:=\left(\begin{array}{cc}
1 & 1 \\
1 & -1
\end{array}\right)
$$

Then we note that

$$
\begin{aligned}
M_{1}\left(\begin{array}{cc}
0 & c(t) \xi \\
c(t) \xi & 0
\end{array}\right) M_{1}^{-1} & =c(t) \xi\left(\begin{array}{cc}
1 & 0 \\
0 & -1
\end{array}\right), \\
M_{1}\left(\begin{array}{cc}
0 & \frac{D_{t} b(t)}{c(t)} \\
0 & \frac{D_{t} c(t)}{c(t)}
\end{array}\right) M_{1}^{-1}= & \frac{D_{t} c(t)}{2 c(t)}\left(\begin{array}{ll}
1 & 0 \\
0 & 1
\end{array}\right) \\
& +\frac{1}{2 c(t)}\left(\begin{array}{cc}
D_{t} b(t) & -D_{t}(b(t)+c(t)) \\
D_{t}(b(t)-c(t)) & -D_{t} b(t)
\end{array}\right),
\end{aligned}
$$

respectively. Thus, after transformation $W_{1}:=M_{1} W_{0}$ the Cauchy problem (3.3) can be written as follows:

$$
\left(D_{t}-\mathcal{D}(t, \xi)-B(t)\right) W_{1}(t, \xi)=0, W_{1}(T, \xi)=M_{1} W_{0}(T, \xi)
$$

where

$$
\mathcal{D}=\mathcal{D}(t, \xi):=\left(\begin{array}{cc}
\tau_{+}(t, \xi) & 0 \\
0 & \tau_{-}(t, \xi)
\end{array}\right), \quad \tau_{ \pm}=\tau_{ \pm}(t, \xi):= \pm c(t) \xi+\frac{D_{t} c(t)}{2 c(t)}
$$

and

$$
B=B(t):=\frac{1}{2 c(t)}\left(\begin{array}{cc}
D_{t} b(t) & -D_{t}(b(t)+c(t)) \\
D_{t}(b(t)-c(t)) & -D_{t} b(t)
\end{array}\right) .
$$


Let $N$ be a positive constant which will be chosen later. To a given $\xi \in \mathbb{R}$ we define $t_{\xi}$ by

$$
t_{\xi}\langle\xi\rangle=N\left(\log t_{\xi}^{-1}\right)^{\gamma} \text { for }|\xi| \geq M \quad \text { and } \quad M \text { large, }
$$

where $\langle\xi\rangle=\sqrt{1+|\xi|^{2}}$, and $\gamma$ is taken from the assumptions of the theorem. We denote the set $\left\{(t, \xi): t \in\left[t_{\xi}, T\right],|\xi| \geq M\right\}$ as the hyperbolic zone $Z_{\text {hyp }}(N, M)$.

REMARK 7. We will prove the statement of Theorem 1 by considerations in $Z_{\text {hyp }}(N, M)$. In the other parts $\{(t, \xi) \in[0, T] \times\{|\xi|<M\}\}$ and in the pseudodifferential zone $Z_{p d}(N, M):=\left\{(t, \xi): t \in\left[0, t_{\xi}\right),|\xi| \geq M\right\}$ we have at most a finite loss of derivatives. For $Z_{p d}(N, M)$ this follows from (3.1) and (3.5) after setting $V(t, \xi):=\left(D_{t} v(t, \xi), \xi v(t, \xi)\right)^{T}$

$$
\begin{gathered}
\left(D_{t}+\left(\begin{array}{cc}
2 b(t) \xi & -a(t)^{2} \xi \\
-\xi & 0
\end{array}\right)\right) V(t, \xi)=0, V(T, \xi)=\left(\begin{array}{c}
-i \hat{\psi}(\xi) \\
\xi \hat{\varphi}(\xi)
\end{array}\right), \\
\|V(t, \cdot)\| \leq \exp \left(C\langle\xi\rangle t_{\xi}\right)\left\|v\left(t_{\xi}, \cdot\right)\right\| \leq\langle\xi\rangle^{C_{N}}\left\|V\left(t_{\xi}, \cdot\right)\right\| \text { for } \gamma \in[0,1] .
\end{gathered}
$$

Let us carry out the second step of diagonalization procedure in $Z_{h y p}(N, M)$. We define $M_{2}=M_{2}(t, \xi)$ by

$$
M_{2}(t, \xi):=\left(\begin{array}{cc}
1 & \frac{B_{12}}{\tau_{-}-\tau_{+}} \\
\frac{B_{21}}{\tau_{+}-\tau_{-}} & 1
\end{array}\right),
$$

where $B_{j k}$ denotes the $(j, k)$-th element of $B$. Then we easily see that $M_{2}$ is invertible in $Z_{\text {hyp }}(N, M)$ for large $N$. Some computations yield

$$
\begin{aligned}
M_{2}^{-1}\left(\left[\mathcal{D}, M_{2}\right]+B M_{2}-\left(D_{t} M_{2}\right)\right) & \\
= & M_{2}^{-1}\left(\left(\begin{array}{cc}
B_{11} & 0 \\
0 & B_{22}
\end{array}\right)+\left(\begin{array}{cc}
\frac{B_{12} B_{21}}{\tau_{+}-\tau_{-}} & \frac{B_{11} B_{12}}{\tau_{-}-\tau_{+}} \\
\frac{B_{21} B_{22}}{\tau_{+}-\tau_{-}} & \frac{B_{12} B_{21}}{\tau_{-}-\tau_{+}}
\end{array}\right)-\left(D_{t} M_{2}\right)\right) \\
= & \left(\begin{array}{cc}
B_{11} & 0 \\
0 & B_{22}
\end{array}\right) \\
& +M_{2}^{-1}\left(\left(I-M_{2}\right)\left(\begin{array}{cc}
B_{11} & 0 \\
0 & B_{22}
\end{array}\right)+\left(\begin{array}{cc}
\frac{B_{12} B_{21}}{\tau_{+}-\tau_{-}} & \frac{B_{11} B_{12}}{\tau_{-}-\tau_{+}} \\
\frac{B_{21} B_{22}}{\tau_{+}-\tau_{-}} & \frac{B_{12} B_{21}}{\tau_{-}-\tau_{+}}
\end{array}\right)-\left(D_{t} M_{2}\right)\right),
\end{aligned}
$$

here we denote the first and the second matrices by $\Phi=\Phi(t, \xi)$, and $B_{2}(t, \xi)$ respectively. Setting $W_{2}(t, \xi):=M_{2}^{-1} W_{1}(t, \xi)$ transforms the Cauchy problem (3.4) to

$$
\left(D_{t}-\mathcal{D}-\Phi-B_{2}\right) W_{2}(t, \xi)=0, \quad W_{2}(T, \xi)=M_{2}(T, \xi)^{-1} W_{1}(T, \xi) .
$$


Finally, we define

$$
M_{3}=M_{3}(t, \xi):=\left(\begin{array}{cc}
\exp \left(-i \int_{t}^{T} \tau_{+}(s, \xi) d s\right) & 0 \\
0 & \exp \left(-i \int_{t}^{T} \tau_{-}(s, \xi) d s\right)
\end{array}\right)
$$

Here we note that $M_{3}^{-1} D_{t} M_{3}=\mathcal{D}$. Thus the transformation $W_{3}:=M_{3}^{-1} W_{2}$ transfers (3.6) to

$$
\left(D_{t}-\Phi-B_{3}\right) W_{3}(t, \xi)=0, W_{3}(T, \xi)=W_{2}(T, \xi),
$$

where $B_{3}:=M_{3}^{-1} B_{2} M_{3}$.

Now let $\theta=\theta(t, \xi) \in L_{1, l o c}\left(\left(t_{\xi}, T\right) ; L_{\infty}(\mathbb{R})\right)$ and define $\Theta=\Theta(t, \xi)$ by

$$
\Theta(t, \xi):=\exp \left(\int_{t}^{T} \theta(s, \xi) d s\right) .
$$

Choosing

$$
Y(t, \xi):=\left(\begin{array}{cc}
\Theta(t, \xi)^{-1} & 0 \\
0 & \Theta(t, \xi)
\end{array}\right) W_{3}(t, \xi)
$$

allows to rewrite the Cauchy problem (3.7) in the form

$$
\begin{gathered}
\left(\partial_{t}-\left(\frac{b^{\prime}(t)}{2 c(t)}+\theta(t, \xi)\right)\left(\begin{array}{cc}
1 & 0 \\
0 & -1
\end{array}\right)-Q(t, \xi)\right) Y(t, \xi)=0 \\
Y(T, \xi)=W_{3}(T, \xi)
\end{gathered}
$$

where

$$
Q=Q(t, \xi):=i\left(\begin{array}{cc}
B_{3,11}(t, \xi) & \Theta(t, \xi)^{-2} B_{3,12}(t, \xi) \\
\Theta(t, \xi)^{2} B_{3,21}(t, \xi) & B_{3,22}(t, \xi)
\end{array}\right)
$$

and $B_{3, j k}$ denotes the $(j, k)$-th element of $B_{3}$. Here we can check that the following estimate holds:

$$
\max _{j, k=1,2}\left\{\left|B_{3, j k}(t, \xi)\right|\right\} \leq r_{1}\langle\xi\rangle^{-1}\left(t^{-1}\left(\log t^{-1}\right)^{\gamma}\right)^{2}
$$

with a positive constant $r_{1}$ in $Z_{h y p}(N, M)$ (for more details we refer to [5], [7], [10]).

Our last transformation yields the reduction to the suitable first order system we wanted to get in this step. 


\section{2. - Lyapunov- and energy function}

We define the Lyapunov function $S=S(t, \xi)$ and the energy function $E=E(t, \xi)$ by

(3.9) $S(t, \xi):=-\left|y_{1}(t, \xi)\right|^{2}+\left|y_{2}(t, \xi)\right|^{2}$ and $E(t, \xi):=\left|y_{1}(t, \xi)\right|^{2}+\left|y_{2}(t, \xi)\right|^{2}$, where $\left(y_{1}, y_{2}\right)=Y^{T}$. Then we can estimate

$$
\begin{aligned}
\partial_{t} S(t, \xi)= & -2 \operatorname{Re}\left(y_{1} \overline{\partial_{t} y_{1}}\right)+2 \operatorname{Re}\left(y_{2} \overline{\partial_{t} y_{2}}\right) \\
= & -2\left(\frac{b^{\prime}}{2 c}+\theta\right) E(t, \xi)+2 \operatorname{Re}\left(y_{1}\left(\overline{Q_{11} y_{1}+Q_{12} y_{2}}\right)\right) \\
& +2 \operatorname{Re}\left(y_{2}\left(\overline{Q_{22} y_{2}+Q_{21} y_{1}}\right)\right) \\
= & -2\left(\frac{b^{\prime}}{2 c}+\theta\right) E(t, \xi)+2 \operatorname{Re}\left(Q_{11}\right)\left|y_{1}\right|^{2}+2 \operatorname{Re}\left(Q_{22}\right)\left|y_{2}\right|^{2} \\
& +2 \operatorname{Re}\left(Q_{12} y_{1} \bar{y}_{2}\right)+2 \operatorname{Re}\left(Q_{21} \overline{y_{1}} y_{2}\right) \\
\leq & -2\left(\frac{b^{\prime}}{2 c}+\theta-2 r_{1} \max \left\{\Theta^{2}, \Theta^{-2}\right\}\langle\xi\rangle^{-1}\left(t^{-1}\left(\log t^{-1}\right)^{\gamma}\right)^{2}\right) E(t, \xi) .
\end{aligned}
$$

Let us define $\zeta(t, \xi)$ by

$$
\begin{aligned}
\zeta(t, \xi):= & \frac{b^{\prime}(t)}{2 c(t)}+\theta(t, \xi) \\
& -2 r_{1} \max \left\{\Theta(t, \xi)^{2}, \Theta(t, \xi)^{-2}\right\}\langle\xi\rangle^{-1}\left(t^{-1}\left(\log t^{-1}\right)^{\gamma}\right)^{2} .
\end{aligned}
$$

If $\zeta(t, \xi) \geq 0$, then the last inequality yields

$$
\partial_{t} S(t, \xi) \leq-2 \zeta(t, \xi) S(t, \xi)
$$

Consequently,

$$
S(T, \xi) \exp \left(\int_{t}^{T} 2 \zeta(s, \xi) d s\right) \leq S(t, \xi) \text { for all } t \in\left[t_{\xi}, T\right] .
$$

In the next steps we have to explain the definition of $\theta$ such that $\zeta(t, \xi) \geq 0$ is really satisfied. Therefore we have to choose the coefficients $a$ and $b$ in a suitable way. This will be done in the next step.

\section{3. - Construction of the coefficients $a(t)$ and $b(t)$}

We define the monotone decreasing sequence of positive real numbers $\left\{t_{j}\right\}_{j=1}^{\infty}$ by

$$
t_{j}:=\exp \left(-j^{\frac{1}{\gamma+1}}\right), \quad j=1,2, \ldots,
$$


and the sequence $\left\{d_{j}\right\}_{j=1}^{\infty}$ by

$$
d_{j}:=\frac{t_{j-1}-t_{j}}{4}, \quad j=1,2, \ldots
$$

Here $t_{0}=T$. Using the mean value theorem we obtain

$$
\sigma_{0}: t_{j}:\left(\log t_{j}^{-1}\right)^{-\gamma} \leq t_{j-1}-t_{j} \leq \sigma_{1} t_{j}\left(\log t_{j}^{-1}\right)^{-\gamma}
$$

where the positive constants $\sigma_{0}, \sigma_{1}$ are independent of $j$. Let us define the coefficients $a=a(t)$ and $b=b(t)$ as follows

$$
a(t):=\int_{t_{j}}^{t} \int_{t_{j}}^{s_{1}} \chi_{j}\left(s_{2}\right) d s_{2} d s_{1}+2 \text { for } t \in\left[t_{j}, t_{j-1}\right), \quad j=1,2, \ldots
$$

and

$$
b(t):= \begin{cases}a\left(t+d_{j}\right) & \text { for } t \in\left[t_{j}, t_{j}+3 d_{j}\right] \\ 2 & \text { for } t \in\left(t_{j}+3 d_{j},: t_{j-1}\right),\end{cases}
$$

where

$$
\chi_{j}(t):= \begin{cases}0 & \text { for } t \in\left[t_{j}, t_{j}+d_{j}\right], \\ -32 d_{j}^{-3}\left(t-\left(t_{j}+d_{j}\right)\right) & \text { for } t \in\left(t_{j}+d_{j}, t_{j}+\frac{5}{4}: d_{j}\right], \\ 32 d_{j}^{-3}\left(t-\left(t_{j}+\frac{3}{2} d_{j}\right)\right) & \text { for } t \in\left(t_{j}+\frac{5}{4} d_{j}, t_{j}+\frac{7}{4}: d_{j}\right], \\ -32 d_{j}^{-3}\left(t-\left(t_{j}+2 d_{j}\right)\right) & \text { for } t \in\left(t_{j}+\frac{7}{4} d_{j}, t_{j}+2 d_{j}\right], \\ 0 & \text { for } t \in\left(t_{j}+2 d_{j}, t_{j}+3 d_{j}\right], \\ 32 d_{j}^{-3}\left(t-\left(t_{j}+3 d_{j}\right)\right) & \text { for } t \in\left(t_{j}+3 d_{j}, t_{j}+\frac{13}{4} d_{j}\right], \\ -32 d_{j}^{-3}\left(t-\left(t_{j}+\frac{7}{2} d_{j}\right)\right) & \text { for } t \in\left(t_{j}+\frac{13}{4} d_{j}, t_{j}+\frac{15}{4}: d_{j}\right], \\ 32 d_{j}^{-3}\left(t-\left(t_{j}+4 d_{j}\right)\right) & \text { for } t \in\left(t_{j}+\frac{15}{4} d_{j}, t_{j-1}\right) .\end{cases}
$$

LEMMA 1. The coefficients $a$ and b given by (3.13) and (3.14) have the following properties:

(i) $a, b \in C^{2}((0, T])$ and for all $j=1,2, \ldots$ it holds

$$
a(t):= \begin{cases}2 & \text { on }\left[t_{j}, t_{j}+d_{j}\right] \\ \text { monotone decreasing } & \text { on }\left(t_{j}+d_{j}, t_{j}+2 d_{j}\right) \\ 1 & \text { on }\left[t_{j}+2 d_{j}, t_{j}+3 d_{j}\right] \\ \text { monotone increasing } & \text { on }\left(t_{j}+3 d_{j}, t_{j-1}\right)\end{cases}
$$


(ii) the constant $R$ from Theorem 1 is given by

$$
R:=8 \sqrt{2} \sigma_{0}^{-1} \sup _{j}\left\{\frac{t_{j}^{-1}\left(\log t_{j}^{-1}\right)^{\gamma}}{t_{j-1}^{-1}\left(\log t_{j-1}^{-1}\right)^{\gamma}}\right\},
$$

more precisely, we have

$$
\max _{t \in\left[t_{j}, t_{j-1}\right]}\left\{\left|a^{\prime}(t)\right|^{2}\right\}=\max _{t \in\left[t_{j}, t_{j-1}\right]}\left\{\left|b^{\prime}(t)\right|^{2}\right\}=2 d_{j}^{-1}
$$

and

$$
\max _{t \in\left[t_{j}, t_{j-1}\right]}\left\{\left|a^{\prime \prime}(t)\right|\right\}=\max _{t \in\left[t_{j}, t_{j-1}\right]}\left\{\left|b^{\prime \prime}(t)\right|\right\}=8 d_{j}^{-2} ;
$$

(iii) there exist positive constants $q_{0}$ and $q_{1}$ independent of $j$ such that

$$
\int_{t_{j}}^{t_{j}+d_{j}} \frac{b^{\prime}(s)}{\sqrt{a(s)^{2}+b(s)^{2}}} d s=-q_{0} ; \quad \int_{t_{j}+2 d_{j}}^{t_{j}+3 d_{j}} \frac{b^{\prime}(s)}{\sqrt{a(s)^{2}+b(s)^{2}}} d s=q_{1},
$$

where $q_{0}<q_{1}$.

Proof. The proof of (i) and (ii) follows from the definitions (3.13) and (3.14) for $a$ and $b$. The relations from (iii) can be checked as follows:

$$
\begin{gathered}
\int_{t_{j}}^{t_{j}+d_{j}} \frac{b^{\prime}(s)}{\sqrt{a(s)^{2}+b(s)^{2}}} d s=\int_{t_{j}}^{t_{j}+d_{j}} \frac{b^{\prime}(s)}{\sqrt{4+b(s)^{2}}} d s=\log \left(\frac{1+\sqrt{5}}{2+2 \sqrt{2}}\right)=:-q_{0}, \\
\int_{t_{j}+2 d_{j}}^{t_{j}+3 d_{j}} \frac{b^{\prime}(s)}{\sqrt{a(s)^{2}+b(s)^{2}}} d s=\int_{t_{j}+2 d_{j}}^{t_{j}+3 d_{j}} \frac{b^{\prime}(s)}{\sqrt{1+b(s)^{2}}} d s=\log \left(\frac{2+\sqrt{5}}{1+\sqrt{2}}\right)=: q_{1},
\end{gathered}
$$

it follows that $q_{0}<q_{1}$.

\section{4. - Construction of $\theta(\mathbf{t}, \xi)$}

From Lemma 1 we can find a positive real number $\delta_{0}$ such that

$$
p_{0}:=\frac{\delta_{0}+q_{0}}{q_{1}} \in(0,1)
$$

We define the sequence of positive functions $\left\{p_{j}=p_{j}(\xi)\right\}_{j \geq 1}$ by

$$
p_{j}(\xi):=K q_{1}^{-1}\langle\xi\rangle^{-1} \int_{t_{j}}^{t_{j-1}}\left(s^{-1}\left(\log s^{-1}\right)^{\gamma}\right)^{2} d s+\frac{q_{0}}{q_{1}}, j=1,2, \ldots,
$$

where $K=e^{2 \delta_{0}}$. Then the condition (3.15) implies that $\sup _{j, \xi}\left\{p_{j}(\xi)\right\} \leq p_{0}$ for large $N$ in (3.5). Indeed, by (3.12) we have

$$
\begin{aligned}
K\langle\xi\rangle^{-1} \int_{t_{j}}^{t_{j-1}}\left(s^{-1}\left(\log s^{-1}\right)^{\gamma}\right)^{2} d s & \leq K\langle\xi\rangle^{-1}\left(t_{j-1}-t_{j}\right)\left(t_{j}^{-1}\left(\log t_{j}^{-1}\right)^{\gamma}\right)^{2} \\
& \leq \frac{K}{N}\left(t_{j-1}-t_{j}\right) t_{j}^{-1}\left(\log t_{j}^{-1}\right)^{\gamma} \leq \frac{\sigma_{1} K}{N} \leq \delta_{0}
\end{aligned}
$$


if we choose $N \geq \sigma_{1} K / \delta_{0}$. By (3.15) we have $\sup _{j, \xi}\left\{p_{j}(\xi)\right\} \leq p_{0}$.

Let us denote $q(t):=b^{\prime}(t) / c(t)$ and introduce as usually the notations

$$
[q(t)]_{+}=\left\{\begin{array}{ll}
q(t) & \text { for } \quad q(t)>0, \\
0 & \text { for } \quad q(t) \leq 0,
\end{array} \text { and }[q(t)]_{-}=\left\{\begin{array}{lll}
0 & \text { for } \quad q(t)>0, \\
q(t) & \text { for } & q(t) \leq 0 .
\end{array}\right.\right.
$$

We note that

$$
\int_{t_{j}}^{t_{j-1}} q(s) d s=-q_{0}+q_{1}
$$

Now let us define

$$
\theta(t, \xi):=-\frac{1}{2}\left(p_{j}(\xi)[q(t)]_{+}+[q(t)]_{-}\right)+\frac{K}{2}\langle\xi\rangle^{-1}\left(t^{-1}\left(\log t^{-1}\right)^{\gamma}\right)^{2}
$$

on $\left[t_{j}, t_{j-1}\right], j=1,2, \ldots$ Then by the assumptions of the theorem, (3.12) and (3.16) we have for $t \in\left[t_{j}, t_{j-1}\right]$ the relations

$$
\begin{aligned}
\left|\int_{t}^{T} \theta(s, \xi) d s\right|= & \left|\int_{t}^{t_{j-1}} \theta(s, \xi) d s+\sum_{k=1}^{j-1} \int_{t_{k}}^{t_{k-1}} \theta(s, \xi) d s\right|=\left|\int_{t}^{t_{j-1}} \theta(s, \xi) d s\right| \\
= & \mid \frac{1}{2} \int_{t}^{t_{j-1}}\left(p_{j}(\xi)[q(s)]_{+}+[q(s)]_{-}\right) d s \\
& -\frac{1}{2} K\langle\xi\rangle^{-1} \int_{t}^{t_{j-1}}\left(s^{-1}\left(\log s^{-1}\right)^{\gamma}\right)^{2} d s \mid \\
\leq & \frac{1}{2} \int_{t}^{t_{j-1}}\left(p_{0}[q(s)]_{+}+[q(s)]_{-}\right) d s \\
& +\frac{1}{2} K\langle\xi\rangle^{-1}\left(t_{j-1}-t_{j}\right)\left(t_{j}^{-1}\left(\log t_{j}^{-1}\right)^{\gamma}\right)^{2} \\
\leq & \frac{1}{2}\left(\delta_{0}+\frac{K \sigma_{1}}{N}\right) \leq \delta_{0} .
\end{aligned}
$$

Therefore we obtain

$$
\max \left\{\Theta(t, \xi)^{2}, \Theta(t, \xi)^{-2}\right\} \leq e^{2 \delta_{0}} .
$$

\section{5. - Properties of $\zeta(t, \xi)$}

Recalling (3.10), (3.16) and (3.17) we have

$$
\begin{aligned}
2 \zeta(t, \xi)= & q(t)-\left(p_{j}(\xi)[q(t)]_{+}+[q(t)]_{-}\right)+K\langle\xi\rangle^{-1}\left(t^{-1}\left(\log t^{-1}\right)^{\gamma}\right)^{2} \\
& -4 r_{1} \frac{K}{4 r_{1}}\langle\xi\rangle^{-1}\left(t^{-1}\left(\log t^{-1}\right)^{\gamma}\right)^{2} \geq\left(1-p_{0}\right)[q(t)]_{+}
\end{aligned}
$$


on $\left[t_{j}, t_{j-1}\right]$. Hence, our assumption $\zeta(t, \xi) \geq 0$ is satisfied. It remains to calculate the integral in the inequality (3.11). We have for $t \in\left[t_{j}, t_{j-1}\right]$ with $j \geq 2$ that

$$
\begin{aligned}
\int_{t}^{T} 2 \zeta(s, \xi) d s & \geq\left(1-p_{0}\right) \sum_{k=1}^{j-1} \int_{t_{k}}^{t_{k-1}}[q(s)]_{+} d s=q_{1}\left(1-p_{0}\right)(j-1) \\
& \geq \frac{q_{1}\left(1-p_{0}\right) j}{2}=\frac{q_{1}\left(1-p_{0}\right)}{2}\left(\log t_{j}^{-1}\right)^{\gamma+1} \geq \frac{q_{1}\left(1-p_{0}\right)}{2}\left(\log t^{-1}\right)^{\gamma+1}
\end{aligned}
$$

by the third statement of Lemma 1 and the definition of $t_{j}$. Choosing in (3.11) $t=t_{j}$ and taking account the last inequality for $t=t_{\xi}$ gives

$$
S(T, \xi) \exp \left(C_{1}\left(\log t_{\xi}^{-1}\right)^{\gamma+1}\right) \leq S\left(t_{\xi}, \xi\right) .
$$

With the definition of $t_{\xi}$ from (3.5) we conclude

$$
\log t_{\xi}^{-1}=\log \langle\xi\rangle-\log N-\gamma \log \left(\log t_{\xi}^{-1}\right) \geq C_{\gamma} \log \langle\xi\rangle-\log N .
$$

Hence, there exist positive constants $C_{1}$ and $C_{N}$ such that for $S(T, \xi) \geq 0$ it holds

$$
C_{N} S(T, \xi) \exp \left(C_{1}(\log \langle\xi\rangle)^{\gamma+1}\right) \leq S\left(t_{\xi}, \xi\right) \text { for }|\xi| \geq M .
$$

\section{6. - Conclusion}

Now we are able to prove the statement of our theorem. Recalling the transformations leading to (3.2), (3.3) and (3.4) we conclude

$$
W_{1}(t, \xi)=e^{-i \xi \int_{t}^{T} b(s) d s}\left(\begin{array}{c}
\left((b(t)+c(t)) \xi+D_{t}\right) v(t, \xi) \\
\left((b(t)-c(t)) \xi+D_{t}\right) v(t, \xi)
\end{array}\right) .
$$

Noting

$$
B_{12}(t)=\frac{-D_{t}(b(t)+c(t))}{2 c(t)}, B_{21}(t)=\frac{D_{t}(b(t)-c(t))}{2 c(t)},\left(\tau_{+}-\tau_{-}\right)(t, \xi)=2 c(t) \xi,
$$

and denoting

$$
m_{1}=m_{1}(t, \xi):=\frac{B_{12}}{\tau_{+}-\tau_{-}}, m_{2}=m_{2}(t, \xi):=\frac{B_{21}}{\tau_{-}-\tau_{+}},
$$

we see that

$$
M_{2}^{-1}=\frac{1}{1+\frac{B_{12} B_{21}}{\left(\tau_{+}-\tau_{-}\right)^{2}}}\left(\begin{array}{cc}
1 & \frac{B_{12}}{\tau_{+}-\tau_{-}} \\
\frac{B_{21}}{\tau_{-}-\tau_{+}} & 1
\end{array}\right)=\frac{1}{1-m_{1} m_{2}}\left(\begin{array}{cc}
1 & m_{1} \\
m_{2} & 1
\end{array}\right) .
$$


The functions $m_{1}$ and $m_{2}$ are pure imaginary and satisfy $\left|m_{k}(t, \xi)\right| \leq \varepsilon, k=1,2$, for any positive small number $\varepsilon$ in $Z_{\text {hyp }}(N, M)$ with sufficiently large $N$. For $W_{2}$ as the solution of (3.6) we get

$$
W_{2}(t, \xi)=\frac{e^{-i \xi \int_{t}^{T} b(s) d s}}{1-m_{1} m_{2}}\left(\begin{array}{c}
\left.\left(b+c+m_{1}(b-c)\right) \xi+\left(1+m_{1}\right) D_{t}\right) v(t, \xi) \\
\left.\left(b-c+m_{1}(b+c)\right) \xi+\left(1+m_{2}\right) D_{t}\right) v(t, \xi)
\end{array}\right) .
$$

Now let us derive an estimate for $\xi^{2}|v(t, \xi)|^{2}+\left|v_{t}(t, \xi)\right|^{2}$.

From (3.18) we conclude

$$
C_{N} S(T, \xi) \exp \left(C_{1}(\log \langle\xi\rangle)^{\gamma+1}\right) \leq\left|y_{1}\left(t_{\xi}, \xi\right)\right|^{2}+\left|y_{2}\left(t_{\xi}, \xi\right)\right|^{2} .
$$

Let us suppose that the a-priori estimate from Theorem 1 holds. Then there exist positive constants $C$ and $s_{1}$ such that for all $(t, \xi) \in[0, T] \times\{|\xi| \geq M\}$ it holds (see [9])

$$
\xi^{2}|v(t, \xi)|^{2}+\left|v_{t}(t, \xi)\right|^{2} \leq C\langle\xi\rangle^{s_{1}}\left(|\xi|^{2}|\varphi(\xi)|^{2}+|\psi(\xi)|^{2}\right),
$$

where $C$ and $s_{1}$ are independent of $\varphi, \psi$. From the above representation for $W_{2}$ and using the transformations to $W_{3}$ and $Y$ with uniformly bounded matrices for all $(t, \xi) \in[0, T] \times\{|\xi| \geq M\}$ we may conclude

$$
\left|y_{1}(t, \xi)\right|^{2}+\left|y_{2}(t, \xi)\right|^{2} \leq C\langle\xi\rangle^{s_{1}}\left(|\xi|^{2}|\varphi(\xi)|^{2}+|\psi(\xi)|^{2}\right) .
$$

Setting $t=t_{\xi}$, the last inequality contradicts (3.19) if e.g. $S(T, \xi) \geq C\langle\xi\rangle^{-s_{2}}$ with a sufficiently large $s_{2}$.

This completes the proof of the theorem.

\section{4. - Finite loss of derivatives}

We can prove the same result as in Theorem 2 in the reverse situation to that is described there, that is, the coefficient $a$ possesses very slow and $b$ possesses fast oscillations in the language of Definition 1.

THEOREM 3. Let us consider the Cauchy problem (1.1). If the coefficients satisfy

- $a, b \in L_{\infty}([0, T]) \cap C^{2}((0, T])$;

- $a(t)^{2}+b(t)^{2} \geq C>0$ on $(0, T]$

- $\left|a^{\prime}(t)\right|^{2}+\left|a^{\prime \prime}(t)\right| \leq\left(R t^{-1}\right)^{2}$ on $(0, T]$;

- $\left|b^{\prime}(t)\right|^{2}+\left|b^{\prime \prime}(t)\right| \leq\left(R t^{-1} \log t^{-1}\right)^{2}$ on $(0, T]$

with a positive constant $R$, then we have the same conclusion as in Theorem 2 . 
ProOF. We explain only the influence of the second integral of (2.3) on the loss of derivatives for $t=t_{\xi}$, where $t_{\xi}$ is defined with $\gamma=1$ as in (3.5) of the proof to Theorem 1 (see also Remark 7). Let us define for $t \in\left[t_{\xi}, T\right]$ the open sets

$$
\Omega_{1}(t)=\left\{\tau \in[t, T]: b^{\prime}(\tau)>0\right\}, \quad \Omega_{2}(t)=\left\{\tau \in[t, T]: b^{\prime}(\tau)<0\right\} .
$$

Using $b \in C^{2}((0, T])$ these sets consist of a countable number of open intervals; thus we can set after introducing suitable notations

$$
\Omega_{1}(t)=\bigcup_{j=1}\left(t_{j}, t_{j-1}\right), \quad \Omega_{2}(t)=\bigcup_{j=1}\left(s_{j}, s_{j-1}\right) .
$$

Let us estimate the integral

$$
\left|\int_{t_{\xi}}^{T} \frac{b^{\prime}(\tau)}{\sqrt{a^{2}(\tau)+b^{2}(\tau)}} d \tau\right| .
$$

If this integral is estimated by $C \log \langle\xi\rangle$ with a constant $C$ independent of $t_{\xi}$, then the loss of regularity of the solution is at most finite. The above representations for $\Omega_{1}(t)$ and $\Omega_{2}(t)$ allow to find sequences of positive real numbers $\left\{p_{j}\right\}$ and $\left\{q_{j}\right\}$ satisfying $p_{j}<q_{j}<p_{j-1}$, with $b^{\prime}(\tau) \leq 0$ on $\left[p_{j}, q_{j}\right]$ and $b^{\prime}(\tau) \geq 0$ on $\left[q_{j}, p_{j-1}\right]$. We define the sequences of real numbers

$$
\begin{aligned}
a_{0, j}: & =\min _{t \in\left[p_{j}, p_{j-1}\right]}\{a(t)\}, a_{1, j}:=\max _{t \in\left[p_{j}, p_{j-1}\right]}\{a(t)\}, \\
b_{0, j}: & =\min _{t \in\left[p_{j}, p_{j-1}\right]}\{b(t)\}, \quad b_{1, j}:=\max _{t \in\left[p_{j}, p_{j-1}\right]}\{b(t)\} .
\end{aligned}
$$

Then there exists a positive constant $r_{1}$ independent of $j$ such that

$$
\begin{aligned}
& a_{1, j}-a_{0, j}=\int_{I_{j}} a^{\prime}(\tau) d \tau, \quad I_{j} \subset\left[p_{j}, p_{j-1}\right], \\
& b_{1, j}-b_{0, j} \leq r_{1}\left(p_{j-1}-p_{j}\right) p_{j}^{-1} \log p_{j}^{-1} \quad \text { (fast oscillations) }
\end{aligned}
$$

if $p_{j}<1$, that is, $j$ large.

From the definition of $p_{j}, q_{j}, p_{j-1}$ we have

$$
\begin{aligned}
\int_{p_{j}}^{q_{j}} \frac{b^{\prime}(\tau)}{\sqrt{a^{2}(\tau)+b^{2}(\tau)} d \tau} & \leq \int_{p_{j}}^{q_{j}} \frac{b^{\prime}(\tau)}{\sqrt{a_{1, j}^{2}+b^{2}(\tau)} d \tau} \\
& =\log \left(\frac{b_{0, j}+\sqrt{a_{1, j}^{2}+b_{0, j}^{2}}}{b_{1, j}+\sqrt{a_{1, j}^{2}+b_{1, j}^{2}}}\right) ; \\
\int_{q_{j}}^{p_{j-1}} \frac{b^{\prime}(\tau)}{\sqrt{a^{2}(\tau)+b^{2}(\tau)} d \tau} & \leq \int_{q_{j}}^{p_{j-1}} \frac{b^{\prime}(\tau)}{\sqrt{a_{0, j}^{2}+b(\tau)^{2}}} d \tau \\
& =\log \left(\frac{b_{1, j}+\sqrt{a_{0, j}^{2}+b_{1, j}^{2}}}{b_{0, j}+\sqrt{a_{0, j}^{2}+b_{0, j}^{2}}}\right)
\end{aligned}
$$


respectively. Let us define

$$
B_{j}(b):=\log \left(\frac{b+\sqrt{a_{1, j}^{2}+b^{2}}}{b+\sqrt{a_{0, j}^{2}+b^{2}}}\right) .
$$

Then we get

$$
\int_{p_{j}}^{p_{j-1}} \frac{b^{\prime}(\tau)}{\sqrt{a(\tau)^{2}+b(\tau)^{2}}} d \tau \leq B_{j}\left(b_{0, j}\right)-B_{j}\left(b_{1, j}\right) .
$$

Applying the mean value theorem there exists a positive constant $C_{b}$ independent of $j$ such that

$$
\begin{aligned}
B_{j}\left(b_{0, j}\right)-B_{j}\left(b_{1, j}\right) & =\left(b_{0, j}-b_{1, j}\right)\left(\frac{1}{\sqrt{a_{1, j}^{2}+b_{2, j}^{2}}}-\frac{1}{\sqrt{a_{0, j}^{2}+b_{2, j}^{2}}}\right) \\
& =\frac{a_{2, j}\left(b_{1, j}-b_{0, j}\right)}{\left(a_{2, j}^{2}+b_{2, j}^{2}\right)^{3 / 2}}\left(a_{1, j}-a_{0, j}\right) \leq C_{b}\left(a_{1, j}-a_{0, j}\right)
\end{aligned}
$$

with some constants $a_{2, j} \in\left[a_{0, j}, a_{1, j}\right]$ and $b_{2, j} \in\left[b_{0, j}, b_{1, j}\right]$. This leads to the estimate

$$
\int_{p_{j}}^{p_{j-1}} \frac{b^{\prime}(\tau)}{\sqrt{a(\tau)^{2}+b(\tau)^{2}}} d \tau \leq C_{b}\left(a_{1, j}-a_{0, j}\right)=C_{b} \int_{I_{j}} a^{\prime}(\tau) d \tau .
$$

It follows that

$$
\int_{t_{\xi}}^{T} \frac{b^{\prime}(\tau)}{\sqrt{a(\tau)^{2}+b(\tau)^{2}}} d \tau \leq C_{b} \int_{t_{\xi}}^{T}\left|a^{\prime}(\tau)\right| d \tau \leq C_{b} R \int_{t_{\xi}}^{T} \tau^{-1} d \tau \leq C_{b, N} R \log \langle\xi\rangle .
$$

This estimate hints to a finite loss of derivatives. Carrying out the second step of diagonalization procedure, estimating the new diagonal matrix and the remainder completes the proof.

Summarizing the statements of our theorems and the previous results, the connection between the classification of the oscillations of the coefficients for (1.1) and the loss of derivatives of the solutions are described in the following Table 1:

TABLE 1: Connection between the classification of the oscillations in the language of Definition 1 and the loss of derivatives

\begin{tabular}{ccccc}
\hline$a(t) \backslash b(t)$ & 0 & very slow & slow & fast \\
\hline 0 & no loss & no loss & small loss & finite loss \\
$\begin{array}{c}\text { very slow } \\
\text { slow }\end{array}$ & no loss & finite loss & finite loss & finite loss \\
fast & finite loss & finite loss & infinite loss & infinite loss
\end{tabular}


REMARK 8. There are special models of type (1.1) for which one can prove the same result as in Proposition 1. If e.g. $b(t)=a(t)$ or more general $a(t)=$ $f(b(t))$, then suitable assumptions for $f$ allow to prove the same connection between type of oscillations and loss of derivatives for $\gamma \in[0,1]$ under the assumption

$$
\left|b^{\prime}(t)\right|^{2}+\left|b^{\prime \prime}(t)\right| \leq\left(R t^{-1}\left(\log t^{-1}\right)^{\gamma}\right)^{2} \quad \text { on } \quad(0, T]
$$

\section{5. - Concluding remarks}

REMARK 9. We can generalize the statement of Theorem 1 to weakly hyperbolic Cauchy problems.

COROLlaRY 1. Let us consider the weakly hyperbolic Cauchy problem

$$
\left(D_{t}^{2}+2 b(t) \lambda(t) D_{x t}^{2}-a(t)^{2} \lambda(t)^{2} D_{x}^{2}\right) u=0, u(T, x)=\varphi(x), u_{t}(T, x)=\psi(x),
$$

where $\lambda(t)=t^{l}, l>0$. There exist coefficients $a, b$ with the same properties as in Theorem 1 such that the energy inequality

$$
\left\|\left(\lambda(t) \partial_{x} u, \partial_{t} u\right)(t, \cdot)\right\|_{H^{s-s_{0}}} \leq C\left\|\left(\varphi^{\prime}(\cdot), \psi(\cdot)\right)\right\|_{H^{s}} \text { for } 0 \leq t<T
$$

does not hold for any positive $s_{0}$ with a positive constant $C$ independent of the data $(\varphi, \psi)$.

SOME REMARKS TO THE PROOF. We follow the proof to Theorem 1. The transformation

$$
w(t, \xi):=\exp \left(-i \xi \int_{t}^{T} b(s) \lambda(s) d s\right) v(t, \xi)
$$

generates the Cauchy problem

$$
\left(D_{t}^{2}-c(t)^{2} \xi^{2}-D_{t}(b(t) \lambda(t)) \xi\right) w(t, \xi)=0 \quad \text { with data on } t=T
$$

where $c(t)=\sqrt{a(t)^{2}+b(t)^{2}} \lambda(t)$. All diagonalization steps are carried out in $Z_{\text {hyp }}(N, M)$ which is defined by $\left\{(t, \xi): t \in\left[t_{\xi}, T\right],|\xi| \geq M\right\}$, where $t_{\xi}$ is implicitly defined by $\Lambda\left(t_{\xi}\right)\langle\xi\rangle=N\left(\log \Lambda\left(t_{\xi}\right)^{-1}\right)^{\gamma}, \Lambda(t)=\int_{0}^{t} \lambda(s) d s$. Using corresponding transformations to those ones from Section 3.1 gives

$$
\begin{gathered}
\left(\partial_{t}-\left(\frac{b^{\prime}(t) \lambda(t)+b(t) \lambda^{\prime}(t)}{2 c(t)}+\theta(t, \xi)\right)\left(\begin{array}{cc}
1 & 0 \\
0 & -1
\end{array}\right)-Q(t, \xi)\right) Y(t, \xi)=0 \\
\text { with datum on } t=T .
\end{gathered}
$$


The property $\lambda^{\prime}(t)>0$ for $t>0$ and the fact that $\exp \left(\int_{t_{\xi}}^{T} \frac{b(s) \lambda^{\prime}(s)}{2 c(s)} d s\right)$ produces at most a finite loss of derivatives make it possible to follow all steps of the proof to Theorem 1. Consequently, a similar estimate to (3.18) holds.

Remark 10. Let us come back to the Cauchy problem (5.1). This arises after application of the partial Fourier transformation with respect to $x$ to

$$
\begin{gathered}
\left(\partial_{t}^{2}-c(t)^{2} \partial_{x}^{2}-\partial_{t}(b(t) \lambda(t)) \partial_{x}\right) u(t, x)=0, \\
u(T, x)=\varphi(x), \quad u_{t}(T, x)=b(T) \lambda(T) \varphi^{\prime}(x)+\psi(x) .
\end{gathered}
$$

If we apply the statement of Theorem 1 to (5.2), then the next result follows immediately.

CoROLlary 2. Let us consider the Cauchy problem $\left(\partial_{t}^{2}-h(t)^{2} \lambda(t)^{2} \partial_{x}^{2}-\partial_{t}(b(t) \lambda(t)) \partial_{x}\right) u(t, x)=0, u(T, x)=\varphi(x), u_{t}(T, x)=\psi(x)$, where $\lambda(t)=t^{l}, l \geq 0$. There exist functions $h$ and $b$ from $C^{2}((0, T])$ which satisfy the conditions

- $h_{0} \leq h(t) \leq h_{1}, b_{0} \leq b(t) \leq b_{1}$;

- $\left|h^{\prime}(t)\right|^{2}+\left|h^{\prime \prime}(t)\right|+\left|b^{\prime}(t)\right|^{2}+\left|b^{\prime \prime}(t)\right| \leq\left(R t^{-1}\left(\log t^{-1}\right)^{\gamma}\right)^{2}$

with positive constants $h_{0}, h_{1}, b_{0}, b_{1}, R$ and with an arbitrary small positive $\gamma$ such that the energy inequality

$$
\left\|\left(\lambda(t) \partial_{x} u, \partial_{t} u\right)(t, \cdot)\right\|_{H^{s-s_{0}}} \leq C\left\|\left(\varphi^{\prime}(\cdot), \psi(\cdot)\right)\right\|_{H^{s}}
$$

does not hold for any positive $s_{0}$ with a positive constant $C$ independent of the data $(\varphi, \psi)$.

This explains the necessity of the Levi condition of $C^{\infty}$-type $\partial_{t}(b(t) \lambda(t))=$ $O\left(\lambda^{\prime}(t)\right)$ in the weakly hyperbolic case $\lambda(t)=t^{l}, l>0$. In the non-Lipschitz case $\lambda(t) \equiv 1$ we get $\partial_{t} b(t)=O\left(t^{-1}\right)$ as a necessary condition. But in opposite to the condition $\partial_{t}(b(t) \lambda(t))=O\left(\lambda^{\prime}(t)\right)$ which is sufficient, too, the condition $\partial_{t} b(t)=O\left(t^{-1}\right)$ seems to be not sufficient. One should suppose the stronger assumption of integrability.

\section{REFERENCES}

[1] R. Agliardi - M. Cicognani, Operators of p-evolution with non regular coefficients in the time variable, J. Differential Equations 202 (2004), 143-157.

[2] M. Cicognani, Coefficients with unbounded derivatives in hyperbolic equations, to appear in Math. Nachr.

[3] F. Colombini - E. De Giorgi - S. Spagnolo, Sur les equations hyperboliques avec des coefficients qui ne dependent que du temps, Ann. Scuola Norm. Sup. Pisa Cl. Sci. (4) 6 (1979), 511-559. 
[4] F. Colombini - D. Del Santo - T. Kinoshita, Well-posedness of the Cauchy problem for a hyperbolic equation with non-Lipschitz coefficients, Ann. Scuola Norm. Sup. Pisa Cl. Sci. (5) 1 (2002), 327-358.

[5] F. Colombini - D. Del Santo -M. Reissig, On the optimal regularity of coefficients in hyperbolic Cauchy problems, Bull. Sci. Math. 127 (2003), 328-347.

[6] F. COLOMbini - N. LeRner, Hyperbolic operators with non-Lipschitz coefficients, Duke Math. J. 77 (1995), 657-698.

[7] F. Hirosawa, On the Cauchy problem for second order strictly hyperbolic equations with non-regular coefficients, Math. Nachr. 256 (2003), 29-47.

[8] F. Hirosawa - M. Reissig, Well-posedness in Sobolev spaces for second order strictly hyperbolic equations with non-differentiable oscillating coefficients, Ann. Global Anal. Geom. 25 (2004), 99-119.

[9] S. Mizonata, "Theory of the partial differential equations", Cambridge University Press, 1973.

[10] M. ReIssIG, Hyperbolic equations with non-Lipschitz coefficients, Rend. Sem. Mat. Univ. Politec. Torino 61 (2003), 135-182.

[11] M. ReISSIG - K. YAGDJIAN, $L_{p}-L_{q}$ estimates for the solutions of strictly hyperbolic equations of second order with increasing in time coefficients, Math. Nachr. 214 (2000), 71-104.

[12] K. Yagdjian, "The Cauchy Problem for Hyperbolic Operators. Multiple Characteristics, Micro-Local Approach”, Akademie-Verlag, Berlin, 1997.

[13] T. YAMAZAKI, On the $L^{2}\left(\mathbb{R}^{n}\right)$ well-posedness of some singular or degenerate partial differential equations of hyperbolic type, Comm. Partial Differential Equations 15 (1990), 1029-1078.

Department of Mathematics Nippon Institute of Technology Saitama 345-8501, Japan hirosawa@nit.ac.jp

Fakultät für Mathematik und Informatik TU Bergakademie Freiberg 09596 Freiberg, Germany reissig@math.tu-freiberg.de 